Physics International 2 (1): 25-30, 2011

ISSN 1948-9803

(C) 2011 Science Publications

\title{
Variable Separation and Boubaker Polynomial Expansion Scheme for Solving the Neutron Transport Equation
}

\author{
${ }^{1}$ Dada, O.M., ${ }^{1}$ O.B. Awojoyogbe, ${ }^{1}$ M. Agida and ${ }^{2}$ K. Boubaker \\ ${ }^{1}$ Department of Physics, \\ Federal University of Technology, Minna, Niger State, Nigeria \\ ${ }^{2}$ Ecole Superieure des Sciences et Techniques de Tunis/63, \\ Rue Sidi Jabeur 5100, Mahdia, Tunisia
}

\begin{abstract}
Problem statement: In this study, we present general analytical solutions to the Neutron Boltzmann Transport Equation NBTE using a polynomial expansion scheme. Approach: Some simple assumptions have been introduced in the main system thanks to the Boubaker Polynomial Expansion Scheme (BPES) in order to make the general analytical procedure simple and adaptable for solving similar real life problems. Results: Finding particular solution to the Neutron equation by making use of boundary conditions and initial conditions may be too much for the present study and reduce the generality of the solutions. Conclusion: The proposed analytical solution of the neutron transport equation has been positively compared to some recently publish results. I should present a relevant supply to studies on reactor modeling.
\end{abstract}

Key words: Neutron transport equation, distribution function, Boubaker Polynomial Expansion Scheme (BPES), source function, neutron angular flux, analytical solutions, seems appropriate, describing neutron, boltzmann equation

\section{INTRODUCTION}

The deterministic neutron transport equation (or Boltzmann neutron transport equation) describes the expected or probable neutron angular density with respect to position, direction, energy and time. Is solutions generally provide average values of the neutron angular density which takes into account random effects from neutron interactions and sources. Since neutrons are neutral particles which possess mass, they can penetrate deep into matter in a nondestructive way despite having characteristics strong forces, a quantum mechanical description seems appropriate, leading to an involved system of Schrödinger equations describing neutron motion between and within nuclei (Kulikowska, 2000; Lewis and Miller, 1984; Bell and Glasstone, 1970; Stammler and Abbate, 1983; Singh, et al., 2010; Nourazar et al., 2011).

From a theoretical point of view, a neutron is a neutral point particle, experiencing deflection from or capture by a nucleus at the center of an atom. If the conditions are just right, the captured neutron causes a fissile nucleus to fission, producing more neutrons. A stochastic partial differential equation is hence derived from these events of neutron transport in a general three-dimensional absorbing and anisotropic-scattering medium where the neutron angular density depends on position, direction, energy and time (Lewis and Miller,
1984). Sometimes, the nucleus that remains as fission product coincides with one of the stable nuclei. The product nucleus is then different from other nuclei, the reason being that the product nucleus is not stable. It disintegrates further, with a mean life characteristic of the nucleus, by emission of an electric charge, until it finally reaches a stable state.

In the present investigation, the medium is assumed to be constant with respect to material composition, i.e., zero power noise. Special random effects, for example, from randomly varying boundary conditions or from a medium that is randomly varying are not considered in the present investigation although generalizations to approximate such conditions may be possible.

\section{MATERIALS AND METHODS}

The neutron transport equation models the transport of neutral particles in a scattering, fission and absorption set of events with no self-interactions (Lewis and Miller, 1984; Chandrasekhar, 1960; Davison, 1957).

In a neutron scattering event (Fig. 1), the quantity to be determined is called the partial differential cross section related to $\Delta \Omega$. This function depends on the energy and momentum transferred from the neutron to the sample. In the most general case the partial differential cross section is a function of four variables since the momentum transfer is a vector quantity with

(C) 2011 Dada, O.M., O.B. Awojoyogbe, M. Agida and K. Boubaker. This open access article is distributed under a Creative Commons Attribution (CC-BY) 3.0 license which permits unrestricted use, distribution, and reproduction in any medium, provided the original work is properly credited. DOI: 10.3844/pisp.2011.25.30 
three components. In elastic scattering, the neutron does not transfer any energy to the sample. The elastic differential cross section is consequently a function of momentum transfer only. From the number of neutrons counted in a detector, the mean values for variables $\theta$ and $\phi$ can be defined (Fig. 1). $\theta$ is the angle through which the neutron has been scattered, i.e. the angle between the incident and the scattered beam.

The paths of the neutrons which are scattered through $\theta$ form a cone so a second (azimuthal) angle, $\phi$, is also needed to define the detector position. Several analytical and numerical approaches have been used in order to solve the neutron transport equation. Jaffel and Vidal-Madjar (1989); Case and Hazeltine (1970) and Davies (2002) performed dicretizing protocols based on Fourier transform, while Cardona and Vilhena (1994); Kim and Ishimaru (1999); Kim and Moscoso (2002); Boyd (2001); Bernardi and Maday (1992) and Kadem (2008) used polynomial expansion schemes..

In the actual investigation, the first assumption is that all particles including nuclei are in motion with like particle collisions allowed, as stated by Kulikowska (2000); Mokhtar-Kharroubbi (1997) and Kadem (2006). The govening equation are Eq. 1:

$$
\begin{aligned}
& \left\{\frac{1}{\mathrm{v}} \frac{\partial}{\partial \mathrm{t}}+\Omega \cdot \nabla+\Sigma(\mathrm{r}, \mathrm{E}, \mathrm{t})\right\} \psi(\mathrm{r}, \Omega, \mathrm{E}, \mathrm{t})= \\
& \int_{0}^{\infty} \mathrm{d} \mathrm{E}^{\prime} \int_{4 \pi} \mathrm{d} \Omega^{\prime} \Sigma_{\mathrm{s}}\left(\mathrm{r}, \Omega^{\prime}, \Omega, \mathrm{E}^{\prime} \rightarrow \mathrm{E}\right) \psi\left(\mathrm{r}, \Omega^{\prime}, \mathrm{E}^{\prime}, \mathrm{t}\right) \\
& +\frac{\chi(\mathrm{E})}{4 \pi} \int_{0}^{\infty} \mathrm{dE}^{\prime} \int_{4 \pi} \mathrm{d} \Omega^{\prime} \mathrm{V}_{\mathrm{E}}\left(\mathrm{E}^{\prime}\right) \Sigma_{\mathrm{f}}\left(\mathrm{r}, \mathrm{E}^{\prime}, \mathrm{t}\right) \psi \\
& \left(\mathrm{r}, \Omega^{\prime}, \mathrm{E}^{\prime}, \mathrm{t}\right)=\mathrm{Q}(\mathrm{r}, \Omega, \mathrm{E}, \mathrm{t})
\end{aligned}
$$

With:

$\mathrm{V}=$ The neutron speed

$\Sigma, \Sigma_{\mathrm{f}} \quad=$ Macroscopic cross-sections

$\Sigma_{\mathrm{s}} \quad=$ Scattering cross-section

$\chi(\mathrm{E}) \quad=$ The distribution function

$\psi=$ The neutron angular flux

E, $\mathrm{E}^{\prime} \quad=$ Energies

$\Omega, \Omega{ }^{\prime} \quad=$ Neutron directions

$\mathrm{Q}(\mathrm{r}, \Omega, \mathrm{E}, \mathrm{t})=$ The source function (Kulikowska, 2000)

Which give, using variable separation Eq. 2:

$$
\begin{aligned}
& \frac{\psi_{2}}{\mathrm{v}} \frac{\partial \psi_{1}}{\partial \mathrm{t}}+\Omega \psi_{2} . \nabla \psi_{1}+\Sigma_{1} \Sigma_{2} \Sigma_{3} \psi_{1} \psi_{2}=\psi_{1} \Sigma_{\mathrm{s} 1}(\mathrm{r}) \\
& \int_{0}^{\infty} \mathrm{dE}^{\prime} \int_{4 \pi} \mathrm{d} \Omega^{\prime} \Sigma_{\mathrm{s} 2}\left(\Omega^{\prime} . \Omega, \mathrm{E}^{\prime} \rightarrow \mathrm{E}\right) \psi_{2}^{\prime}+\frac{\chi(\mathrm{E})}{4 \pi} . \\
& \psi_{1} \psi_{2}^{\prime} \int_{0}^{\infty} \mathrm{d} \mathrm{E}^{\prime} \int_{4 \pi} \mathrm{d} \Omega^{\prime} \mathrm{V}_{\mathrm{E}}\left(\mathrm{E}^{\prime}\right) \Sigma_{\mathrm{f} 1} \Sigma_{\mathrm{f} 2} \Sigma_{\mathrm{f} 3}+\mathrm{Q}(\mathrm{r}, \Omega, \mathrm{E}, \mathrm{t})
\end{aligned}
$$

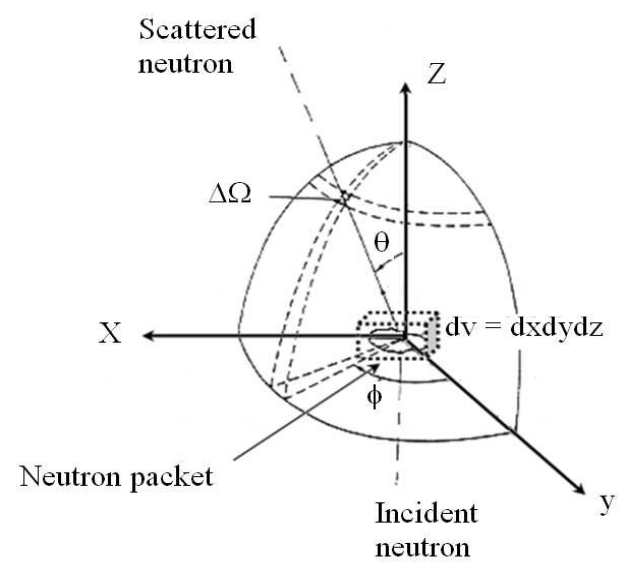

Fig. 1: Neutron scattering event geometrical model which gives Eq. 3:

$$
\begin{aligned}
& \frac{\psi_{2}}{\mathrm{v}} \frac{\partial \psi_{1}}{\partial \mathrm{t}}+\Omega \psi_{2} \frac{\partial \psi_{1}}{\partial \mathrm{r}}+ \\
& \left\{\begin{array}{l}
\Sigma_{1} \Sigma_{2} \Sigma_{3} \psi_{2}-\psi_{2}^{\prime} \Sigma_{\mathrm{s} 1}(\mathrm{r}) \\
\int_{0}^{\infty} \mathrm{d} \mathrm{E}^{\prime} \int_{4 \pi} \mathrm{d} \Omega^{\prime} \Sigma_{\mathrm{s} 2}-\frac{\chi(\mathrm{E})}{4 \pi} \cdot \psi_{2}^{\prime} \Sigma_{\mathrm{f} 1}(\mathrm{r}) \Sigma_{\mathrm{f} 3} \\
(\mathrm{t}) \int_{0}^{\infty} \mathrm{V}_{\mathrm{E}}\left(\mathrm{E}^{\prime}\right) \Sigma_{\mathrm{f} 2}\left(\mathrm{E}^{\prime}\right) \mathrm{d \textrm {E } ^ { \prime }} \int_{4 \pi} \mathrm{d} \Omega^{\prime}
\end{array}\right\} \psi_{1}=\mathrm{Q}
\end{aligned}
$$

where, $V_{E}$ represents the average number of neutrons per fission.

By setting Eq. 4:

$$
\begin{aligned}
& \psi_{1}(\mathrm{r}, \mathrm{t})=\mathrm{R}(\mathrm{r}) \mathrm{G}(\mathrm{t}) \\
& \mathrm{A}(\mathrm{r}, \mathrm{t})=\mathrm{A}_{1}(\mathrm{r})+\mathrm{A}_{2}(\mathrm{t}) \\
& \mathrm{Q}_{1}(\mathrm{r}, \mathrm{t})=\psi_{1} \mathrm{Q}_{0} \\
& \mathrm{Q}_{0}=\mathrm{Q}_{0}(\mathrm{r})+\mathrm{G}_{0}(\mathrm{t})
\end{aligned}
$$

One obtains:

$$
\begin{gathered}
\frac{\Psi_{2}}{\mathrm{Q}_{2} \mathrm{v}} \mathrm{R} \frac{\mathrm{dG}}{\mathrm{dt}}+\frac{\Omega \psi_{2}}{\mathrm{Q}_{2}} \mathrm{G} \frac{\mathrm{dR}}{\mathrm{dr}}+\left\{\frac{\mathrm{A}_{1}(\mathrm{r})+\mathrm{A}_{2}(\mathrm{t})}{\mathrm{Q}_{2}}\right\} \\
\mathrm{RG}=\mathrm{RG}\left(\mathrm{R}_{0}+\mathrm{G}_{0}\right) \frac{\Psi_{2}}{\mathrm{Q}_{2} \mathrm{v}} \frac{1}{\mathrm{G}} \frac{\mathrm{dG}}{\mathrm{dt}}+\frac{\Omega \Psi_{2}}{\mathrm{Q}_{2}} \frac{1}{\mathrm{R}} \frac{\mathrm{dR}}{\mathrm{dr}} \\
+\frac{\mathrm{A}_{1}(\mathrm{r})}{\mathrm{Q}_{2}}+\frac{\mathrm{A}_{2}(\mathrm{t})}{\mathrm{Q}_{2}}=\left(\mathrm{R}_{0}+\mathrm{G}_{0}\right) \frac{\Psi_{2}}{\mathrm{Q}_{2} \mathrm{v}} \frac{1}{\mathrm{G}} \frac{\mathrm{dG}}{\mathrm{dt}}+ \\
\mathrm{Z} \frac{\Omega \psi_{2}}{\mathrm{Q}_{2}} \frac{1}{\mathrm{R}} \frac{\mathrm{dR}}{\mathrm{dr}}+\frac{\mathrm{A}_{1}(\mathrm{r})}{\mathrm{Q}_{2}}+\frac{\mathrm{A}_{2}(\mathrm{t})}{\mathrm{Q}_{2}}=\left(\mathrm{R}_{0}+\mathrm{G}_{0}\right) \\
\frac{\psi_{2}}{\mathrm{Q}_{2} \mathrm{v}} \frac{1}{\mathrm{G}} \frac{\mathrm{dG}}{\mathrm{dt}}+\frac{\mathrm{A}_{2}(\mathrm{t})}{\mathrm{Q}_{2}}-\mathrm{G}_{0}(\mathrm{t})=- \\
\frac{\Omega \Psi_{2}}{\mathrm{Q}_{2}} \frac{1}{\mathrm{R}} \frac{\mathrm{dR}}{\mathrm{dr}}-\frac{\mathrm{A}_{1}(\mathrm{r})}{\mathrm{Q}_{2}}+\mathrm{R}_{0}(\mathrm{r})
\end{gathered}
$$


Phy. Intl. 2 (1): 25-30, 2011

Since both sides of Eq. 5 are independent of one another, they must be equal to a constant $\varepsilon^{2}$; leading to the following equations Eq. 6 and 7:

$$
\begin{aligned}
& \frac{\mathrm{dG}}{\mathrm{dt}}=\frac{\mathrm{Q}_{2} \mathrm{v}}{\Psi_{2}}\left\{\mathrm{G}_{0}(\mathrm{t})-\frac{\mathrm{A}_{2}(\mathrm{t})}{\mathrm{Q}_{2}}+\varepsilon^{2}\right\} \mathrm{G}(\mathrm{t}) \\
& \frac{\mathrm{dR}}{\mathrm{dr}}=\frac{\mathrm{Q}_{2}}{\Omega \psi_{2}}\left\{\mathrm{R}_{0}(\mathrm{r})+\varepsilon^{2}-\frac{\mathrm{A}_{1}(\mathrm{r})}{\mathrm{Q}_{2}}\right\} \mathrm{R}(\mathrm{r})
\end{aligned}
$$

which have as solutions Eq. 8 and 9:

$G(t)=P_{0} e^{\frac{Q_{2} v}{\psi_{2}} \int\left\{G_{0}(t)-\frac{A_{2}(t)}{Q_{2}}+\varepsilon^{2}\right\}} d t$

$\mathrm{R}(\mathrm{r})=\mathrm{P}_{1} \mathrm{e}^{\frac{\mathrm{Q}_{2}}{\Omega \psi_{2}} \int\left\{\mathrm{R}_{0}(\mathrm{r})+\varepsilon^{2}-\frac{\mathrm{A}_{1}(\mathrm{r})}{\mathrm{Q}_{2}}\right\} \mathrm{dr}}$

where, $\mathrm{P}_{0}$ and $\mathrm{P}_{1}$ are constants.

The expression for the flux is hence given as Eq. 10:

$$
\left\{\begin{array}{l}
\psi(\mathrm{r}, \Omega, \mathrm{E}, \mathrm{t})=\mathrm{P}_{0} \mathrm{P}_{1} \psi_{2}(\Omega, \mathrm{E}) \mathrm{e}^{\frac{\mathrm{Q}_{2}}{\psi_{2}}\left(\mu_{1}+\mu_{2}\right)} \\
\mu_{1}=\mathrm{v} \int\left\{\mathrm{G}_{0}(\mathrm{t})-\frac{\mathrm{A}_{2}(\mathrm{t})}{\mathrm{Q}_{2}}+\varepsilon^{2}\right\} \mathrm{dt} \\
\mu_{2}=\frac{1}{\Omega} \int\left\{\mathrm{R}_{0}(\mathrm{r})+\varepsilon^{2}-\frac{\mathrm{A}_{1}(\mathrm{r})}{\mathrm{Q}_{2}}\right\} \mathrm{dr}
\end{array}\right.
$$

\section{RESULTS}

Different expressions to $\mathrm{Q}_{2}, \mathrm{G}_{0}, \mathrm{~A}_{2}, \mathrm{~A}_{1}$ and $\mathrm{R}_{\mathrm{o}}$ allow obtaining different expressions of the macroscopic cross section which is generally expressed as Eq. 11:

$$
\Sigma_{\mathrm{ij}}(\mathrm{r}, \mathrm{E}, \mathrm{t})=\mathrm{N}_{\mathrm{j}}(\mathrm{r}, \mathrm{t}) \sigma_{\mathrm{ij}}(\mathrm{E})
$$

For a given nuclide $\mathrm{j}$ and reaction type $\mathrm{i}$, where $\mathrm{N}_{\mathrm{j}}$ $(r, t)$ is the nuclear atomic density and $\sigma_{i j}(E)$ is the microscopic cross section, then, if we assume that nuclear atomic density is independent of time Eq. 12 and 13:

$$
\Sigma_{\mathrm{ij}}=\mathrm{N}_{\mathrm{j}}(\mathrm{r}) \sigma_{\mathrm{ij}}(\mathrm{E})
$$

Then:

$$
\left\{\begin{array}{l}
\Sigma_{3}(\mathrm{t})=1 \\
\Sigma(\mathrm{r}, \mathrm{E})=\Sigma_{1}(\mathrm{r}) \Sigma_{2}(\mathrm{E})
\end{array}\right.
$$

And the expression for the distribution function $\chi($ E) becomes (Kulikowska, 2000; Kadem, 2006; Mokhtar-Kharroubbi, 1997) Eq. 14:

$$
\begin{gathered}
\frac{\chi(\mathrm{E})}{4 \pi} \psi_{2}^{\prime} \Sigma_{\mathrm{f} 1}(\mathrm{r}) \int_{0}^{\infty} \mathrm{V}_{\mathrm{E}}\left(\mathrm{E}^{\prime}\right) \Sigma_{\mathrm{f} 2}\left(\mathrm{E}^{\prime}\right) \mathrm{d} \mathrm{E}^{\prime} \int_{4 \pi} \mathrm{d} \Omega^{\prime}=\Sigma_{1}(\mathrm{r}) \Sigma_{2}(\mathrm{E}) \\
\psi_{2}-\mathrm{A}(\mathrm{r})-\psi_{2}^{\prime} \Sigma_{\mathrm{s} 1}(\mathrm{r}) \int_{0}^{\infty} \Sigma_{\mathrm{sb}}\left(\mathrm{E}^{\prime} \rightarrow \mathrm{E}\right) \mathrm{d} \mathrm{E}^{\prime} \int_{4 \pi} \Sigma_{\mathrm{sa}}\left(\Omega^{\prime} \Omega\right) \mathrm{d} \Omega^{\prime} \\
4 \pi \Sigma_{1}(\mathrm{r}) \Sigma_{2}(\mathrm{E}) \psi_{2}-4 \pi \mathrm{A}(\mathrm{r})-4 \pi \psi_{2}^{\prime} \Sigma_{\mathrm{s} 1} \\
\chi(\mathrm{E})=\frac{(\mathrm{r}) \int_{0}^{\infty} \Sigma_{\mathrm{sb}}\left(\mathrm{E}^{\prime} \rightarrow \mathrm{E}\right) \mathrm{dE^{ \prime }} \int_{4 \pi} \Sigma_{\mathrm{sa}}\left(\Omega^{\prime} \Omega\right) \mathrm{d} \Omega^{\prime}}{\psi_{2}^{\prime} \Sigma_{\mathrm{f} 1}(\mathrm{r}) \int_{0}^{\infty} \mathrm{V}_{\mathrm{E}}\left(\mathrm{E}^{\prime}\right) \Sigma_{\mathrm{f} 2}\left(\mathrm{E}^{\prime}\right) \mathrm{d} \mathrm{E}^{\prime} \int_{4 \pi} \mathrm{d} \Omega^{\prime}}
\end{gathered}
$$

\section{DISCUSSION}

Criticality and analytical solutions can be discussed by considering the special case of the neutron transport equation without delayed neutrons, which is traduced by the equation:

$$
\begin{aligned}
& \left\{\frac{1}{\mathrm{v}} \frac{\partial}{\partial \mathrm{t}}+\Omega \nabla+\sigma(\overrightarrow{\mathrm{r}}, \mathrm{E})\right\} \psi(\overrightarrow{\mathrm{r}}, \Omega, \mathrm{E}, \mathrm{t})=\mathrm{Q}_{\mathrm{ext}}(\overrightarrow{\mathrm{r}}, \Omega, \mathrm{E}, \mathrm{t}) \\
& +\int \mathrm{d} \mathrm{E}^{\prime} \int \mathrm{d} \Omega^{\prime} \sigma_{\mathrm{s}}\left(\overrightarrow{\mathrm{r}}, \Omega^{\prime} . \Omega, \mathrm{E}^{\prime} \rightarrow \mathrm{E}\right) \psi\left(\overrightarrow{\mathrm{r}}, \Omega, \mathrm{E}^{\prime}, \mathrm{t}\right) \\
& +\chi(\mathrm{E}) \int \mathrm{d} \mathrm{E}^{\prime} \int \mathrm{d} \Omega^{\prime} \mathrm{V}_{\mathrm{E}} \sigma_{\mathrm{f}}\left(\overrightarrow{\mathrm{r}}, \mathrm{E}^{\prime}\right) \psi\left(\overrightarrow{\mathrm{r}}, \Omega^{\prime}, \mathrm{E}^{\prime}, \mathrm{t}\right)
\end{aligned}
$$

With:

$\mathrm{Q}_{\mathrm{ext}}=$ The external sources of neutrons

$\sigma=$ The microscopic cross-section

$\mathrm{v}=$ The neutron speed

$\mathrm{V}_{\mathrm{E}}=$ Average number of neutrons per fission

This equation assumes that all neutrons are emitted instantaneously at the time of fission although small fraction of neutrons is emitted later due to certain fission products.

If we look for an asymptotic solutions to Eq. 15, satisfying the source free boundary conditions $\left(\mathrm{Q}_{\mathrm{ext}}=\right.$ $0)$, it gives:

$$
\begin{array}{r}
\mathrm{B}(\overrightarrow{\mathrm{r}})=\frac{\sigma_{\mathrm{s} 1}(\overrightarrow{\mathrm{r}})}{\psi_{\mathrm{a} 3}(\mathrm{E})} \int \sigma_{\mathrm{s} 2}\left(\mathrm{E}^{\prime} \rightarrow \mathrm{E}\right) \psi_{\mathrm{a} 3}^{\prime}\left(\mathrm{E}^{\prime}\right) \mathrm{dE}^{\prime} \\
\int \sigma_{\mathrm{s} 3}\left(\Omega^{\prime} \Omega\right) \psi_{\mathrm{a} 2} \mathrm{~d} \Omega^{\prime}-\frac{\alpha}{\mathrm{v}}-\sigma_{1}(\overrightarrow{\mathrm{r}}) \sigma_{2}(\mathrm{E}) \\
+\frac{\chi(\mathrm{E}) \mathrm{V}_{\mathrm{E}}}{\psi_{\mathrm{a} 2}(\Omega) \psi_{\mathrm{a} 3}(\mathrm{E})} \int \sigma_{\mathrm{f} 2}\left(\mathrm{E}^{\prime}\right) \psi_{\mathrm{a} 3}^{\prime}\left(\mathrm{E}^{\prime}\right) \mathrm{d \textrm {E } ^ { \prime }} \\
\int \sigma_{\mathrm{f} 1}(\overrightarrow{\mathrm{r}}) \psi_{\mathrm{a} 2}^{\prime}\left(\Omega^{\prime}\right) \mathrm{d} \Omega^{\prime}
\end{array}
$$

And:

$$
\Omega \frac{\mathrm{d} \psi_{\mathrm{a} 1}}{\mathrm{dr}}=\mathrm{B}(\overrightarrow{\mathrm{r}}) \psi_{\mathrm{a} 1}
$$


Phy. Intl. 2 (1): 25-30, 2011

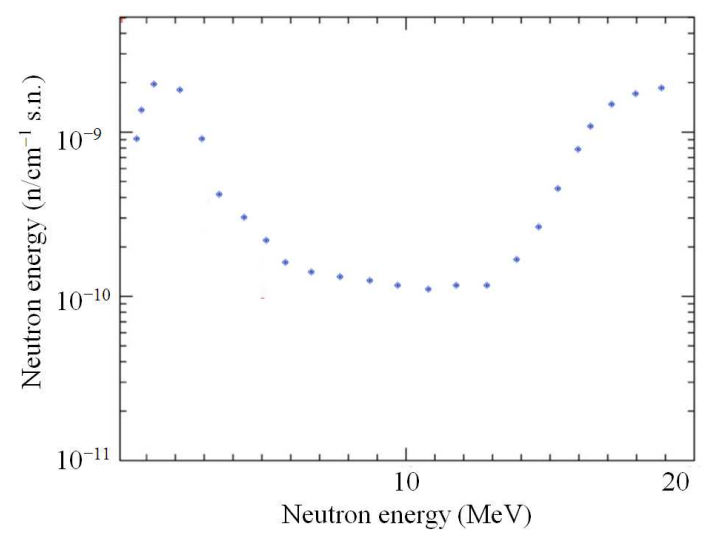

Fig. 2: Energy-dependent neutron flux profile

If we suppose that the integral $\int_{0}^{\infty} \frac{B(\vec{r})}{\Omega} d \vec{r}$ is convergent and taking into account the characteristics of a given nuclear reactor with spherical symmetry $\left(\mathrm{B}(\overrightarrow{\mathrm{r}})=\|\mathrm{B}(\overrightarrow{\mathrm{r}})\| \frac{\overrightarrow{\mathrm{r}}}{\|\overrightarrow{\mathrm{r}}\|}=\|\mathrm{B}(\overrightarrow{\mathrm{r}})\| \overrightarrow{\mathrm{u}}_{\mathrm{r}}\right.$ within radial range $[0, \mathrm{R}]$ :

$$
\left\{\begin{array}{l}
\left.\mathrm{B}(\overrightarrow{\mathrm{r}})\right|_{\mathrm{r}=\overline{0}}=\mathrm{k}_{1} \\
\left.\frac{\mathrm{d}\|\mathrm{B}(\overrightarrow{\mathrm{r}})\|}{\mathrm{dr}}\right|_{\mathrm{i}=\overline{0}}=0 \\
\left.\mathrm{~B}(\overrightarrow{\mathrm{r}})\right|_{\mathrm{i}=R \overline{\mathrm{u}}_{r}}=0 \\
\left.\frac{\mathrm{d}\|\mathrm{B}(\overrightarrow{\mathrm{r}})\|}{\mathrm{dr}}\right|_{\overline{\mathrm{r}}=\mathrm{R}_{\mathrm{r}}}=\mathrm{k}_{2}
\end{array}\right.
$$

where, $\mathrm{k}_{1} \mathrm{k}_{2}$ are core reactor characteristic constants.

For solving Eq. 16-17, the Boubaker Polynomials Expansion Scheme BPES is proposed. This scheme is applied through setting the expression:

$$
\|\mathrm{B}(\overrightarrow{\mathrm{r}})\|=\frac{1}{2 \mathrm{~N}_{0}} \sum_{\mathrm{k}=1}^{\mathrm{N}_{0}} \lambda_{\mathrm{k}} \times \mathrm{B}_{4 \mathrm{k}}\left(\frac{\mathrm{r}}{\mathrm{R}} \mu_{\mathrm{k}}\right)
$$

where, $\mathrm{B}_{4 \mathrm{k}}$ are the $4 \mathrm{k}$-order Boubaker polynomials, $\mathrm{r}$ is the radius $\left(r \in[0, R), \mu_{k}\right.$ are $B_{4 k}$ minimal positive roots, $\mathrm{N}_{0}$ is a prefixed integer and $\left.\lambda_{\mathrm{k}}\right|_{\mathrm{k}=1 . . \mathrm{N}_{0}}$ are unknown pondering real coefficients.

At this stage, the main advantage of this step lies in Eq. 19 which ensures verifying the four boundary conditions in Eq. 18, at the earliest stage of resolution protocol. In fact, due to the properties of the Boubaker polynomials (Ghanouchi et al., 2007; Awojoyogbe and Boubaker, 2009; Labiadh and Boubaker, 2007; Slama et al., 2009; Hossein et al., 2009; Fridjine and Amlouk,
2009; Belhadj et al., 2009a; 2009b, Barry and Hennessy, 2010; Agida and Kumar, 2010; Yildirim et al., 2010; Kumar, 2010; Milgram, 2011) and since $\left.\mu_{\mathrm{k}}\right|_{\mathrm{k}=1 . . \mathrm{N}_{0}}$ are roots of $\left.B_{4 k}\right|_{k=1.1 . N_{0}}$, Eq. 15 is reduced to Eq. 20:

$$
\begin{aligned}
& \mid \begin{array}{l}
\sum_{\mathrm{k}=1}^{\mathrm{N}_{0}} \lambda_{\mathrm{k}} \times\left.\mathrm{B}_{4 \mathrm{k}}\left(\frac{\mathrm{r}}{\mathrm{R}} \mu_{\mathrm{k}}\right)\right|_{\mathrm{i}=0}=\sum_{\mathrm{k}=1}^{\mathrm{N}_{0}} \lambda_{\mathrm{k}} \times(-2)=2 \mathrm{k}_{1} \mathrm{~N}_{0} \\
\sum_{\mathrm{k}=1}^{\mathrm{N}_{0}} \lambda_{\mathrm{k}} \times\left.\frac{\mathrm{dB}_{4 \mathrm{k}}\left(\frac{\mathrm{r}}{\mathrm{R}} \mu_{\mathrm{k}}\right)}{\mathrm{dr}}\right|_{\mathrm{r}=\overline{0}}=\sum_{\mathrm{k}=1}^{\mathrm{N}_{0}} \lambda_{\mathrm{k}} \times 0=0 \\
\sum_{\mathrm{k}=1}^{\mathrm{N}_{0}} \lambda_{\mathrm{k}} \times\left.\mathrm{B}_{4 \mathrm{k}}\left(\frac{\mathrm{r}}{\mathrm{R}} \mu_{\mathrm{k}}\right)\right|_{\mathrm{i}=\mathrm{R}_{\mathrm{r}}}=\sum_{\mathrm{k}=1}^{\mathrm{N}_{0}} \lambda_{\mathrm{k}} \times \mathrm{B}_{4 \mathrm{k}}\left(\mu_{\mathrm{k}}\right) \mid=0
\end{array} \\
& \sum_{\mathrm{k}=1}^{\mathrm{N}_{0}} \lambda_{\mathrm{k}} \times\left.\frac{\mathrm{dB}_{4 \mathrm{k}}\left(\frac{\mathrm{r}}{\mathrm{R}} \mu_{\mathrm{k}}\right)}{\mathrm{dr}}\right|_{\bar{r}=\mathrm{R}_{\mathrm{r}}}=\sum_{\mathrm{k}=1}^{\mathrm{N}_{0}} \lambda_{\mathrm{k}} \times \\
& \frac{\mathrm{dB}_{4 \mathrm{k}}\left(\mu_{\mathrm{k}}\right)}{\mathrm{dr}}=\sum_{\mathrm{k}=1}^{\mathrm{N}_{0}} \lambda_{\mathrm{k}} \times \mathrm{H}_{\mathrm{k}}=2 \mathrm{k}_{2} \mathrm{~N}_{0} \\
& \text { with : } \mathrm{H}_{\mathrm{k}}=\left.\frac{\mathrm{dB}_{4 \mathrm{k}}\left(\frac{\mathrm{r}}{\mathrm{R}} \mu_{\mathrm{k}}\right)}{\mathrm{dr}}\right|_{\bar{r}=\mathrm{R}_{\mathrm{r}}}= \\
& \left(\frac{4 \mu_{\mathrm{k}}\left[2-\mu_{\mathrm{k}}^{2}\right] \times \sum_{\mathrm{j}=1}^{\mathrm{k}} \mathrm{B}_{4 \mathrm{j}}^{2}\left(\mu_{\mathrm{k}}\right)}{\mathrm{B}_{4(\mathrm{k}+1)}\left(\mu_{\mathrm{k}}\right)}+4 \mu_{\mathrm{k}}^{3}\right)
\end{aligned}
$$

The solution is then assigned to the set of pondering real coefficients $\left.\tilde{\lambda}_{\mathrm{k}}\right|_{k=1 . . \mathrm{N}_{0}}$ which minimizes the Minimum Square functional $\Psi_{\mathrm{N} 0}$ :

$\Psi_{\mathrm{N}_{0}}=\left(\sum_{\mathrm{k}=1}^{\mathrm{N}_{0}} \tilde{\lambda}_{\mathrm{k}} \times(-2)-2 \mathrm{k}_{1} \mathrm{~N}_{0}\right)^{2}+\left(\sum_{\mathrm{k}=1}^{\mathrm{N}_{0}} \tilde{\lambda}_{\mathrm{k}} \times \mathrm{H}_{\mathrm{k}}=2 \mathrm{k}_{2} \mathrm{~N}_{0}\right)^{2}$

Which gives the following solution to Eq. 21:

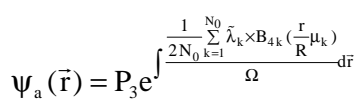

With $\mathrm{P}_{3}$ constant.

From Eq. 22 and our earlier assumptions, we write Eq. 23:

$\psi_{\mathrm{a}}(\overrightarrow{\mathrm{r}}, \Omega, \mathrm{E})=\mathrm{P}_{3} \psi_{\mathrm{a} 2}(\Omega) \Psi_{\mathrm{a} 3}(\mathrm{E}) \int \frac{\frac{1}{2 \mathrm{~N}_{0}} \sum_{\mathrm{k}=1}^{\mathrm{N}_{0}} \tilde{\lambda}_{\mathrm{k}} \times \mathrm{B}_{4 \mathrm{k}}\left(\frac{\mathrm{r}}{\mathrm{R}} \mu_{\mathrm{k}}\right)}{\Omega} \mathrm{dr}$ 
$\psi(\overrightarrow{\mathrm{r}}, \Omega, \mathrm{E}, \mathrm{t})=\mathrm{P}_{3} \psi_{\mathrm{a} 2}(\Omega) \psi_{\mathrm{a} 3}(\mathrm{E}) \mathrm{e}^{\alpha \mathrm{t}+\int \frac{\frac{1}{2 \mathrm{~N}_{0}} \sum_{\mathrm{k}=1}^{N_{0}} \tilde{\lambda}_{\mathrm{k}} \times \mathrm{B}_{4 k}\left(\frac{\mathrm{r}}{\mathrm{R}} \mu_{\mathrm{k}}\right)}{\Omega} \mathrm{d} \overrightarrow{\mathrm{r}}}$

Figure 2 presents the profile of the energydependent neutron flux as per Eq. 24. The obtained patterns are in good agreement with the results recorded by Lent et al. (2004); Zeyad et al. (2007) and Zhao et al. (2006).

\section{CONCLUSION}

An analytical solution of the neutron transport equation applying the Boubaker Polynomial Expansion Scheme BPES has been presented. The solution plots and main features have shown a good agreement with some recently published results and should present a relevant supply to studies on reactor modeling. Effects of power noise, special random effects and variable boundary conditions are subjects of future studies.

\section{REFERENCES}

Agida, M. and A.S. Kumar, 2010. A boubaker polynomials expansion scheme solution to random love's equation in the case of a rational Kernel. Elec. J. Theoretical Phys., 7: 319-326.

Awojoyogbe, O.B. and K. Boubaker, 2009. A solution to Bloch NMR flow equations for the analysis of hemodynamic functions of blood flow system using m-Boubaker polynomials. Curr. Applied Phys., 9: 278-283. DOI: 10.1016/j.cap.2008.01.019

Barry, P. and A. Hennessy, 2010. Meixner-type results for riordan arrays and associated integer sequences. J. Integer Seq., 13: 1-34.

Belhadj, A., J. Bessrour, M. Bouhafs and L. Barrallier, 2009a. Experimental and theoretical cooling velocity profile inside laser welded metals using keyhole approximation and Boubaker polynomials expansion. J. Thermal Analysis Calorimetry, 97: 911-920. DOI: 10.1007/s10973-009-0094-4

Belhadj, A., O.F. Onyango and N. Rozibaeva, 2009b. Boubaker polynomials expansion scheme-related heat transfer investigation inside keyhole model. J. Thermophys Heat Transf., 23: 639-640.

Bell, G.I. and S. Glasstone, 1970. Nuclear Reactor Theory. 1st Edn., Van Nostrand Reinhold Company, New York, pp: 619.

Bernardi, C. and Y. Maday, 1992. Approximations spectrales de problèmes aux limites elliptiques. 1st Edn., Springer-Verlag, Paris, ISBN-10: 3540595767, pp: 242.
Boyd, J.P., 2001. Chebyshev and Fourier Spectral Methods. 2nd Edn. Courier Dover Publications, Mineola, ISBN-10: 0486411834, pp: 668.

Cardona, A.V. and M.T. Vilhena, 1994. A solution of the linear transport equation using Chebyshev polynomials and Laplace transform. Kerntechnik, 59: 278-281.

Case, K.M. and R.D. Hazeltine, 1970. Three-Dimensional Linear Transport Theory. J. Math. Phys., 11: 1126-1135. DOI: $10.1063 / 1.1665238$

Chandrasekhar, S., 1960. Radiative Transfer. 1st Edn., Courier Dover Publications, New York, ISBN-10: 0486605906, pp: 393.

Davies, B., 2002. Integral transforms and their applications. 3rd Edn., Springer, New York, ISBN10: 0387953140 , pp: 367.

Davison, B., 1957. Neutron Transport Theory. 1st Edn., Clarendon Press, London, pp: 450.

Fridjine, S. and M. Amlouk, 2009. A new parameter: An abacus for optimizing pv-t hybrid solar device functional materials using the boubaker polynomials expansion scheme. Modern Phys. Lett., 23: 2179-2191. DOI: $10.1142 / \mathrm{S} 0217984909020321$

Ghanouchi, J., H. Labiadh and K. Boubaker, 2007. An attempt to solve the heat transfert equation in a model of pyrolysis spray using 4q-order m-Boubaker polynomials. Int. J. Heat Technol., 26: 49-53.

Hossein, S.A., A.E. Tabatabaei, T. Zhao, O.B. Awojoyogbe and F.O. Moses, 2009. Cut-off cooling velocity profiling inside a keyhole model using the Boubaker polynomials expansion scheme. Heat Mass Transfer. 45: 1247-1251. DOI: 10.1007/s00231-009-0493-x

Jaffel, L.B. and A. Vidal-Madjar, 1989. New developments in the discrete ordinate method for the resolution of the radiative transfer equation, Astron. Astrophys., 220: 306-312.

Kadem, A., 2006. Analytical solutions for the neutron transport using the spectral methods. Int. J. Math. Math. Sci., 2006: 1-11.

Kadem, A., 2008. Solving transport equation via walsh function and sumudu transform. Appli. Comput. Math., 7: 107-118.

Kim, A.D. and A. Ishimaru, 1999. A chebyshev spectral method for radiative transfer equations applied to electromagnetic wave propagation and scattering in a discrete random medium. J. Comput. Phys., 152: 264-280. DOI: 10.1006/jcph.1999.6247

Kim, A.D. and M. Moscoso, 2002. Chebyshev spectral methods for radiative transfer. SIAM J. Sci. Comput., 23: 2075-2095. 
Kulikowska, T., 2000. An introduction to the neutron transport phenomena. Proceecings of the Lecture given at the Workshop on Nuclear Data and Nuclear Reactors: Physics, design and safety. Mar. 13-14, Trieste, Italy, pp: 1-20.

Kumar, A.S., 2010. An analytical solution to applied mathematics-related Love's equation using the Boubaker polynomials expansion scheme. J. Franklin Inst., 347: 1755-1761. DOI: 10.1016/j.jfranklin.2010.08.008

Labiadh, H. and K. Boubaker, 2007. A Sturm-Liouville shaped characteristic differential equation as a guide to establish a quasi-polynomial expression to the Boubaker polynomials. Elect. J., 2: 117-133.

Lent, E.M., K.E. Sale, R.M. Buck, M. Descalle, 2004. COG validation: SINBAD Benchmark Problems. U.S. Department of Energy.

Lewis, E.E. and W.F. Miller, 1984. Computational Methods of Neutron Transport. 1st Edn., Wiley, New York, ISBN-10: 0471092452, pp: 401

Milgram, A., 2011. The stability of the Boubaker polynomials expansion scheme (BPES)-based solution to Lotka-Volterra problem. J. Theoretical Biol., 271: 157-158. DOI: 10.1016/j.jtbi.2010.12.002

Mokhtar-Kharroubbi, M., 1997. Mathematical Topics in Neutron Transport Theory: New Aspects. 1st Ed. World Scientific Publishing, Singapore, ISBN: 9810228694, pp: 244.

Nourazar, S. S., A. Nazari-Golshan and M. Nourazar, 2011. On the closed form solutions of linear and nonlinear cauchy reaction-diffusion equations using the hybrid of fourier transform and variational iteration method. Phys. Int., 2: 8-20. DOI: $10.3844 /$ pisp.2011.8.20

Singh, V., M.K. Singh and R. Pathak, 2010. Two source emission behaviour of alpha fragments of projectile having energy around $1 \mathrm{GeV}$ per nucleon. Proceedings of the 16th International Symposium on Very High Energy Cosmic Ray Interactions, Jun. 28-Jul. 2, Batavia, IL, USA., pp: 1-4.
Slama, S., J. Bessrour, M. Bouhafs and K.B.B. Mahmoud, 2009. Numerical distribution of temperature as a guide to investigation of melting point maximal front spatial evolution during resistance spot welding using boubaker polynomials. Numer. Heat Tran. Part A: Appli., 55: 401-408. DOI: 10.1080/10407780902720783

Stammler, R.J. J. and M.J. Abbate, 1983. Methods of Steady-State Reactor Physics in Nuclear Design. 1st Edn., Academic Press, London, ISBN-10: 0126633207, pp: 506.

Yildirim, A., S.T. Mohyud-Din and D.H. Zhang, 2010. Analytical solutions to the pulsed Klein-Gordon equation using Modified Variational Iteration Method (MVIM) and Boubaker Polynomials Expansion Scheme (BPES). Comput. Math. Appli., 59: 2473-2477. DOI: 10.1016/j.camwa.2009.12.026

Zeyad A.D. and E.I. Starovoitov, 2007. Physical nonlinearity under cyclic loading in neutron flow. Am. J. Applied Sci., 4: 653-657.

Zhao, Z. and G.I. Maldonado, 2006. Speedup of Particle Transport Problems with a Beowulf Cluster. Am. J. Applied Sci., 3: 1948-1951. DOI:10.3844/ajassp.2006.1948.1951 